\title{
Remarkable transmission of microwaves through a wall of long metallic bricks
}

\author{
Alastair P. Hibbins ${ }^{\text {a) }}$ and J. Roy Sambles \\ Thin Film Photonics Group, School of Physics, University of Exeter, Exeter EX4 4QL, United Kingdom \\ Chris R. Lawrence and Donna M. Robinson \\ QinetiQ (Structures and Materials Centre), Farnborough GU14 OLX, United Kingdom
}

(Received 15 June 2001; accepted for publication 13 August 2001)

\begin{abstract}
The transmitted intensity of a microwave beam through a thick continuous metal wall will be effectively zero due to the almost complete exclusion of the electric field from the metal. However, it is shown here that by removing less than $20 \%$ of the wall material to produce a regular array of bricks, up to $90 \%$ of the radiation is transmitted, despite the gaps between the bricks being less than $5 \%$ of the incident wavelength. This result is attributed to the excitation of a set of resonant waves along the cavity length through the coupling together of surface-plasmon modes across its width. (C) 2001 American Institute of Physics. [DOI: 10.1063/1.1412593]
\end{abstract}

The inspiration for this study originated from the interest in the enhanced transmission of radiation through structured metallic media such as freestanding rough ${ }^{1}$ or corrugated ${ }^{2,3}$ metallic films and periodic arrays of subwavelength holes. ${ }^{4,5}$ These results have been attributed to either resonant or evanescent coupling between the surface-plasmon polaritons ${ }^{6}$ (SPPs) that propagate along both the illuminated and back surfaces. ${ }^{2,7,8}$ Another recent development has been the realization of a family of self-coupled standing wave surface plasmons in deep, metal reflection gratings. ${ }^{9-12}$ In addition, Porto et al. ${ }^{13}$ modeled the response of nondiffracting metallic diffraction gratings of $\left(\sim \lambda_{0}\right)$ thickness with very narrow slits ( $\ll \lambda_{0}, \lambda_{g}$ where $\lambda_{g}$ is the grating pitch). Similar modeling was undertaken by Astilean et al. ${ }^{14}$ who demonstrated the transmission of light through a one-dimensional array of subwavelength width slits in silver films. They showed that, on resonance, the modes are standing waves formed by the coupling together of two SPPs, one on each metal-air interface, across the cavity width. Very recently, the enhanced selective transmission of microwave radiation though a nondiffracting metal-slat grating was experimentally recorded. ${ }^{15}$

In this study we explore the remarkably high resonant transmissivity of microwave radiation through a thick $\left(>\lambda_{0}\right.$, where $\lambda_{0}$ is the free-space wavelength), two-dimensional grating array. A square wooden frame, approximately $450 \mathrm{~mm} \times 450 \mathrm{~mm}$ is constructed, into which a series of grooves, each separated from the next by $4.4 \mathrm{~mm}$, are machined. The groove pattern on each side is mirrored by a pattern on the opposite side. A polymer fiber with a diameter of approximately $0.4 \mathrm{~mm}$ is then wrapped around the frame so that it fits into the grooves. This leads to a regular square grid of fiber at both the front and back faces of the frame into which approximately 8000 aluminum-alloy, square-section rods are inserted (Fig. 1). The resulting sample is effectively a nondiffracting (at normal incidence) wall of long metallic bricks or rods, where each rod is $35.64 \pm 0.06 \mathrm{~mm}$ long and $3.98 \pm 0.01 \mathrm{~mm} \times 3.98 \pm 0.01 \mathrm{~mm}$ in cross section, separated

a)Electronic mail: a.p.hibbins@exeter.ac.uk from its neighbors by a cavity $0.41 \pm 0.02 \mathrm{~mm}$ wide. The size of the array $(385 \mathrm{~mm})$ is substantially bigger than the diameter of the incident microwave beam $(\sim 200 \mathrm{~mm})$, and hence the structure is effectively infinite in extent in the $x-z$ plane.

The arrangement used to study the transmissivity in this work was designed so that a wide parallel microwave beam is incident upon the sample. ${ }^{16}$ The radiation in the wavelength range $7.5<\lambda_{0}<16.7 \mathrm{~mm}$ is linearly polarized. To ac-

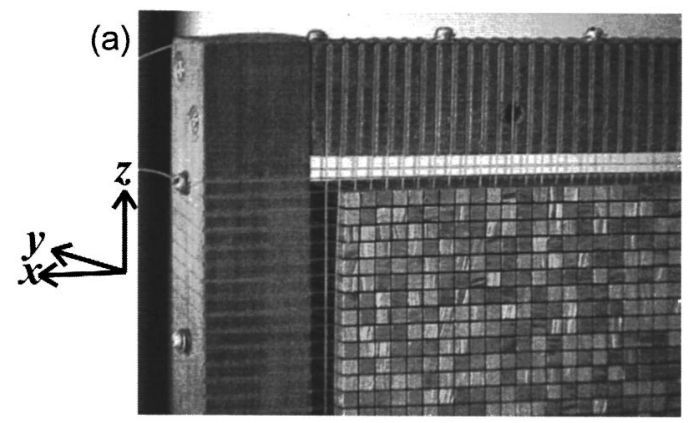

(b)

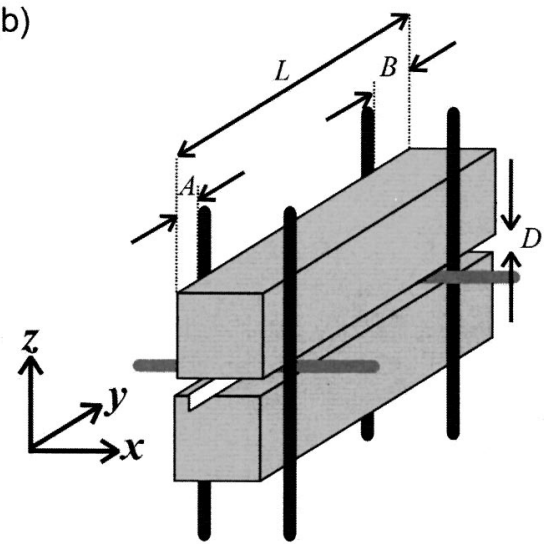

FIG. 1. (a) Photograph of a section of the sample used in this study. The length and width of each rod in the array are approximately 36 and $4 \mathrm{~mm}$, respectively, and the size of the complete sample has side lengths of around $385 \mathrm{~mm}$. (b) Schematic diagram illustrating the geometry of the fiber (black and dark gray) with respect to the metal rods. The white area (that extends along the entire length of the rod) indicates the region over which the fields illustrated in Fig. 3 are calculated. 


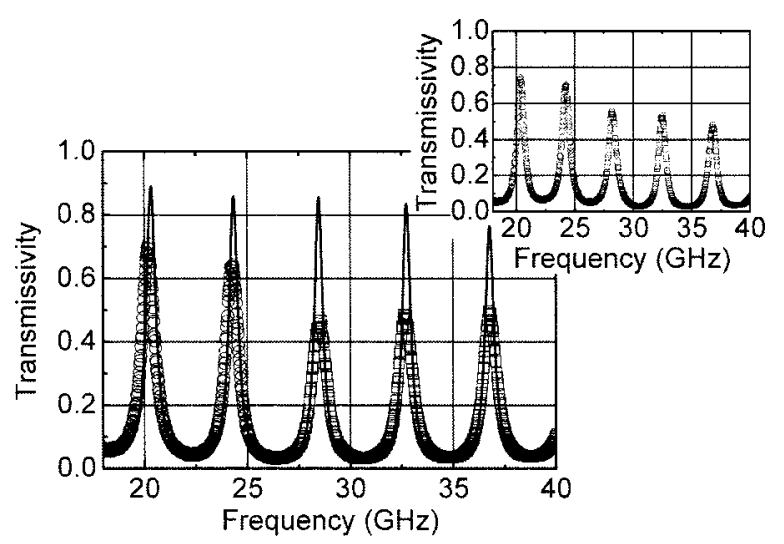

FIG. 2. Experimentally measured transmitted signal through the sample using normally incident radiation polarized with its electric vector orientated in the $x$ direction. The inset shows a near-identical response when the orthogonal polarization is incident. The circles and squares represent the experimentally measured data recorded when using the two different sets of horn antennas and waveguides. The solid line in the main diagram illustrates the best-fit predictions calculated using the theoretical model.

count for any fluctuations in the power emitted from the source, an analyzer divides the output from the signal detector (via a horn antenna) by that from the reference. The resulting transmissivities from the sample are normalized by comparison with the intensity of the beam without the sample.

Figure 2 shows the extraordinary wavelength-dependent transmissivities through the sample when the microwave electric field vector is orientated in the $x$ direction at normal incidence. The response from the sample for the orthogonal polarization is shown in the inset. Notice the remarkable result that the wavelengths at which the transmission maxima are observed are more than 20 times the width of the air cavities. In addition, the transmission efficiencies through the sample are as high as $500 \%$ when normalized to the area of the cavity openings. By using the position of the spacing fibers within the cavities $[A$ and $B$; see Fig. 1(b)] and the refractive index of the fiber $\left(n_{\text {fiber }}\right)$ as fitting parameters, the best-fit numerical model to the experimental data is illustrated as a solid line. This modeled response was obtained by using a code based on a finite element method (Ansoft HFSS) with $A=6.5 \mathrm{~mm}, B=4.6 \mathrm{~mm}$, and $n_{\text {fiber }}=2.4$ (where $A$ and $B$ were allowed to vary within the errors in their measured values, $A=6.2 \pm 0.3 \mathrm{~mm}$ and $B=5.0 \pm 0.5 \mathrm{~mm}$ ). The modeled transmission peaks are clearly more intense than those observed experimentally; this is due to the residual angle spread in the beam. ${ }^{16}$ Since one set of horn antennas and waveguides is required to study the $7.5<\lambda_{0}<11.3 \mathrm{~mm}$ range, and another is required for the $11.3<\lambda_{0}<16.7 \mathrm{~mm}$ range, the degree of beam spread, and hence the peak transmittivities over the two wavelength ranges, will also vary, and this is clearly evident in Fig. 2. It is expected that, at normal incidence, the two linear, orthogonal polarizations would be degenerate and excite equivalent modes, yet small differences are found in both the position and the height of the transmission resonances between the two polarizations (Fig. 2). However upon rotating the sample $90^{\circ}$ the responses to the two incident polarizations are exactly reversed. The original differences in the responses are therefore due to the geometry of the fibers that space the rods [Fig. 1(b)].

Downloaded $24 \mathrm{Feb} 2006$ to 144.173.231.32. Redistribution subject to AIP license or copyright, see http://apl.aip.org/apl/copyright.jsp
The real part of the wave vector of a coupled SPP, $k_{\mathrm{SPP}}$, supported by a narrow open-ended cavity similar to those studied here can be shown to be ${ }^{11}$

$$
k_{\mathrm{SPP}} \approx k_{0}\left[1+\frac{1}{2} \eta^{2}\left(1+\sqrt{1+\frac{4}{\eta^{2}}\left(1+\left|\epsilon_{\text {metal }}\right|\right)}\right)\right] .
$$

In this equation, $\eta=2 /\left(k_{0} D\left|\epsilon_{\text {metal }}\right|\right)$, where $k_{0}=2 \pi / \lambda_{0}$ is the free-space wave vector of the incident beam, $\epsilon_{\text {metal }}$ is the complex permittivity of the metal and $D$ is the cavity width. As predicted by Astilean et al., ${ }^{14}$ the wave vector of the coupled mode $\left(k_{\mathrm{SPP}}\right)$ is expected to depend on the width of the cavity. However, for the microwave study presented in this work, $\left|\epsilon_{\text {metal }}\right|$ is extremely large such that with $D$ $\sim 10^{-4} \mathrm{~m}, \quad \eta$ is very small $\left(\sim 10^{-4}\right)$. Hence, the coupled SPPs will have wave vectors very close to the free-space wave vector $k_{0}$ and will be excited when the incident wavelength satisfies the Fabry-Pérot condition,

$$
\lambda_{0}=\frac{2 n_{\mathrm{eff}} L}{N},
$$

for normal incidence radiation. Here $N$ is the number of nodes (regions of zero field) within the cavity of length $L$ and effective refractive index $n_{\text {eff }}$.

Using the experimentally determined position of the transmission peaks and Eq. (2), the orders of the resonant modes and the value of the effective refractive index of the cavities may be determined. In Fig. 2, the peaks correspond to the $N=5,6,7,8$ and 9 modes and hence the effective refractive index of the cavities is calculated to be $n_{\text {eff }}=1.04$ \pm 0.01 . The uncertainty in this value is an indication of local effects (e.g., the position of the spacing fibers) on the microwave field distribution within the cavities. In order to verify this estimate, the effective refractive index is also calculated in a different way. The value of the refractive index of the fiber determined using the finite element modeling discussed above $\left(n_{\text {fiber }}=2.4\right)$ and the percentage of volume of the fiber within the cavity (2\%) yield the value $n_{\text {eff }}=1.03$ the two estimates are clearly in good agreement.

Computer modeling also gives the microwave electric field intensity within the cavities. This is illustrated both for on and off resonance in Fig. 3 at points in the plane located at the midpoint of the rod's side [Fig. 1(b)] with the incident electric field orientated in the $x$ direction. The regions of high field intensity across the width of the cavity verify that for on resonance of the $20.4 \mathrm{GHz}$ mode, the SPPs on both metalair interfaces couple together across the gap and the magnitude of the electric field within parts of cavity is enhanced by approximately 500\%. For the resonance shown, the cavity contains five minima in electric field intensity (five half wavelengths) which corresponds to the $N=5$ Fabry-Pérot resonance.

The work presented here has demonstrated experimentally that a structurally strong and thick wall of metallic bricks spaced by narrow dielectric-filled cavities may show remarkable transmission of electromagnetic radiation. For specific frequencies, up to $90 \%$ of radiation incident upon the sample may be transmitted. This is despite the fact that the dielectric area presented is less than one fifth the area of the incident beam, and that the gaps between the rods are less than 5\% of the incident wavelength. Since the transmission 
(a)

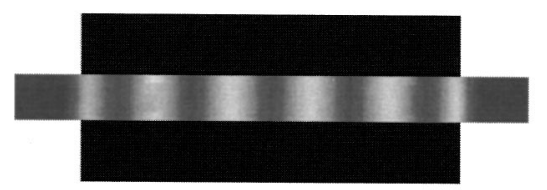

(b)

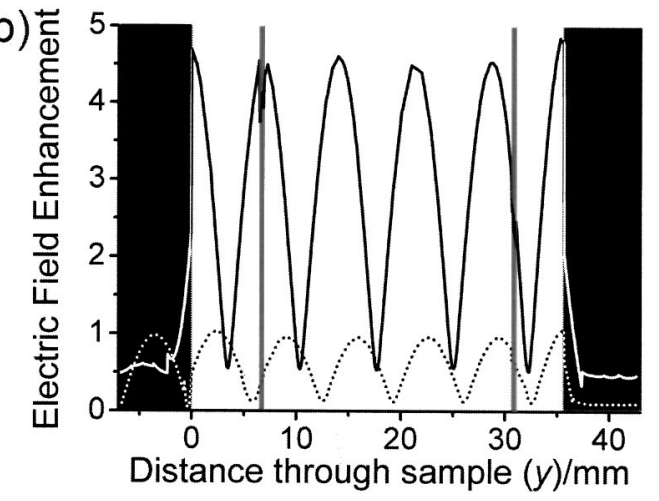

FIG. 3. (a) Calculated electric field intensity in the vicinity of the cavity and in the plane described in Fig. 1(b) on resonance of the $20.3 \mathrm{GHz}$ mode. (b) Line graph showing the on $(20.3 \mathrm{GHz})$ and off resonance $(22.0 \mathrm{GHz})$ electric field enhancement along the center of the cavity (solid and broken lines, respectively). The field enhancement outside the sample is shown in the black regions, and the positions of the line are also illustrated (gray lines).

of radiation relies on a resonant coupling condition being satisfied, the device appears completely opaque at other millimeter wavelengths. Its use as a nondiffractive polarizationinsensitive microwave filter is therefore obvious: the length of the bricks and the refractive index of the material between the bricks govern the frequencies at which it operates. The manufacture of samples scaled down in dimension for use at infrared or even visible frequencies may also have significant technical and commercial potential.

The authors would like to thank Piers Andrew and Pete Cann for their help in constructing the sample. This work was carried out as part of Technology Group 09 of the MoD Corporate Research Program.

${ }^{1}$ J. A. Sanchez-Gil, A. A. Maradudin, J. Q. Lu, and V. D. Freilikher, Phys. Rev. B 51, 17100 (1995).

${ }^{2}$ U. Schroter and D. Heitmann, Phys. Rev. B 58, 15419 (1998).

${ }^{3}$ I. Avrutsky, Y. Zhao, and V. Kochergin, Opt. Lett. 25, 595 (2000).

${ }^{4}$ T. W. Ebbesen, H. J. Lezec, H. F. Ghamei, T. Thio, and P. A. Wolff, Nature (London) 391, 667 (1998).

${ }^{5}$ T. Thio, H. F. Ghaemi, H. J. Lezec, P. A. Wolff, and T. W. Ebbesen, J. Opt. Soc. Am. B 16, 1743 (1999).

${ }^{6} \mathrm{H}$ Raether, Surface Plasmons (Springer, Berlin, 1988).

${ }^{7}$ R. Sambles, Nature (London) 391, 641 (1998).

${ }^{8}$ L. Martín-Moreno, F. J. García-Vidal, H. J. Lezec, K. M. Pellerin, T. Thio, T. J. B. Pendry, and T. W. Ebbesen, Phys. Rev. Lett. 86, 1114 (2001).

${ }^{9}$ F. J. García-Vidal and J. B. Pendry, Phys. Rev. Lett. 77, 1163 (1996).

${ }^{10}$ F. J. García-Vidal, J. Sánchez-Dehesa, A. Dechelette, E. Bustarret, T. López-Ríos, T. Fournier, and B. Pannetier, J. Lightwave Technol. 17, 2191 (1999).

${ }^{11}$ M. B. Sobnack, W. C. Tan, N. P. Wanstall, T. W. Preist, and J. R. Sambles, Phys. Rev. Lett. 80, 5667 (1998).

${ }^{12}$ W. C. Tan, T. W. Preist, J. R. Sambles, and N. P. Wanstall, Phys. Rev. B 59, 12661 (1999).

${ }^{13}$ J. A. Porto, F. J. García-Vidal, and J. B. Pendry, Phys. Rev. Lett. 83, 2845 (1999).

${ }^{14}$ S. Astilean, P. Lalanne, and M. Palamaru, Opt. Commun. 175, 265 (2000).

${ }^{15}$ H. E. Went, A. P. Hibbins, J. R. Sambles, C. R. Lawrence, and A. P. Crick, Appl. Phys. Lett. 77, 2789 (2000).

${ }^{16}$ A. P. Hibbins, J. R. Sambles, and C. R. Lawrence, J. Appl. Phys. 86, 1791 (1999). 